\title{
Visibility of CT Early Ischemic Change Is Significantly Associated with Time from Stroke Onset to Baseline Scan beyond the First 3 Hours of Stroke Onset
}

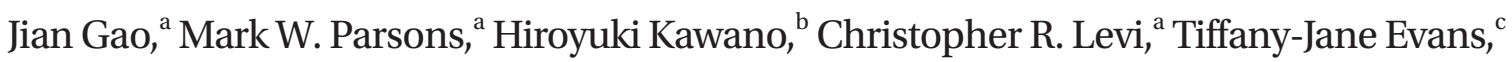 \\ Longting Lin, ${ }^{a}$ Andrew Bivard ${ }^{\mathrm{a}}$ \\ ${ }^{a}$ Department of Neurology, John Hunter Hospital, University of Newcastle, Newcastle, New South Wales, Australia \\ ${ }^{b}$ Department of Stroke and Cerebrovascular Medicine, Kyorin University, Mitake, Tokyo, Japan \\ 'Centre for Bioinformatics, Hunter Medical Research Institute, Newcastle, New South Wales, Australia
}

Background and Purpose Non-contrast brain computed tomography (NCCT) remains the most common imaging modality employed to select patients for thrombolytic therapy in acute ischemic stroke. The current study used the Alberta Stroke Program Early CT Score (ASPECTS) to identify early ischemic changes on brain NCCT imaging with the aim to investigate whether a relationship exists between time from symptoms onset to NCCT with the presence of early ischaemic change quantified by ASPECTS.

Methods We studied 1,329 ischemic stroke patients who had NCCT within 8 hours of stroke onset. Patients were assessed to see if they had any ASPECTS lesion and if the rate of patients with a lesion increased with time using logistic regression.

Results 30\% patients had an ASPECTS $<10$ within the first 3 hours from symptom onset. Within the first 3 hours, the odds for a CT change (ASPECTS $<10$ ) per minute of time was 1.00 with 95\% confidence interval $(\mathrm{Cl})(0.99$ to 1.00$)(P=0.266)$. After 3 hours, there was a significant increase in odds of ASPECTS $<10$ with increasing time. The odds of being ASPECTS positive increased 1\% (odds ratio=1.01) per 1 minute of time with $95 \% \mathrm{Cl}(1.00$ to 1.01$)(P=0.002)$.

Conclusions We have identified that prior to first 3 hours of stroke there was no effect of time on odds of CT ischemic change; after the first 3 hours of stroke the odds increased with increasing time to CT scan. The occurrence of early ischemic change may be a marker of time from stroke onset rather than severity.

Keywords Stroke; Tomography, X-ray computed; Time
Correspondence: Andrew Bivard Department of Neurology, John Hunter Hospital, University of Newcastle, 1 Kookaburra Circuit, New Lambton Heights, NSW 2305, Australia

Tel: +61-2-4042-0315

Fax: +61-2-4042-0001

E-mail: andrew.bivard@newcastle.edu.au

Received: September 23, 2016

Revised: June 8, 2017

Accepted: August 13, 2017

The authors have no financial conflicts of interest.

\section{Introduction}

In acute ischemic stroke, despite alternative imaging techniques becoming increasingly available, head non-contrast computed tomography (NCCT) remains the current standard of care imaging for intravenous thrombolysis selection. ${ }^{1-3}$ Pooled analyses of the
National Institute of Neurological Disorders and Stroke (NINDS) rtPA Stroke Study, ${ }^{4}$ the European Cooperative Acute Stroke Studies (ECASS), ${ }_{1}^{5-7}$ and the Alteplase Thrombolysis for Acute Noninterventional Therapy in Ischemic Stroke (ATLANTIS) trials ${ }^{8,9}$ have identified that alteplase therapy in patients screened using NCCT resulted in improved patient outcomes especially within the first 3 hours 
of stroke. ${ }^{3}$

Brain NCCT is not only used to exclude patients with hemorrhage, but also to assess potential suitability for reperfusion therapy. One sign of early ischemic change (EIC) seen on NCCT is widespread hypo-attenuation ( $>1 / 3$ cerebral hemisphere), which is a recognized predictor of neurological deterioration after intravenous thrombolysis treatment. ${ }^{1,3}$ However, the results from the Multicenter Randomized Clinical Trial of Endovascular Treatment for Acute Ischemic Stroke in the Netherlands (MR CLEAN) study were contrary to findings from previous trials. ${ }^{10}$ The subgroup analysis of MR CLEAN suggested that extent of EIC seen on NCCT (the Alberta Stroke Program Early CT Score, ASPECTS 0-7) within the first 6 hours of stroke was not correlated with a poorer outcome. Thus, it was suggested that patients with ASPECTS 0-7 might yield similar benefit, compared with patients with ASPECTS $>7$, from intra-arterial treatment.

The prognostic value of NCCT for acute infarction may be low because the appearance of hypo-attenuation is time-dependent. ${ }^{4,11}$ A $1 \%$ increase in brain water content corresponds to an approximate change of 1.8-2.6 hounsfield units $(\mathrm{HU})$. $^{11,12}$ In acute infarction, due to the degradation of brain blood barrier, cerebral tissue continuously takes up water from residual blood flow as time progresses. ${ }^{13,14}$ Previous animal studies have identified that middle cerebral artery occlusion leads to $0.7-1.3 \%$ increase in the concentration of water in primates within the first 2 hours of stroke, $^{15}$ as well as a $2 \%$ increase in water content in a cat model of stroke within 4 hours. ${ }^{11,12,16}$ Therefore, the ischemic brain parenchyma during the first 3 hours of stroke would lead to an approximate 2-4 HU decrease on NCCT, which is the lowest HU difference detected by human visual inspection. ${ }^{17-19}$ After the first 3 hours of stroke, the increase in water content of tissue due to infarction will be within the visible spectrum of $>2-4 \mathrm{HU}$. We aimed to investigate the relationship between symptom onset time and EIC detection using ASPECTS score in a cohort of acute ischemic stroke patients. We hypothesized that there would be a significant relationship between time and ASPECTS score.

\section{Methods}

\section{Study participants}

Clinical and imaging information from acute ischemic stroke patients presenting to hospital within 8 hours of symptom onset at 13 centers in Australia, China, Canada, and India between 2011-2015 were prospectively collected for the International Stroke Perfusion Imaging Registry (INSPIRE). These sites were involved in the registry as they routinely perform multimodal CT prior to reperfusion therapy. The imaging information was baseline multimodal CT (NCCT and CTA), and follow-up imaging at 24-48 hours post-stroke. All patients in the current study had an intracranial occlusion. Patients with severe motion artifacts which rendered the imaging unreadable were excluded from the INSPIRE study due to the registry design. Clinical stroke severity was assessed at the two imaging time points using the National Institutes of Health Stroke Scale (NIHSS). Clinical information included time from symptom onset to imaging and treatment. Patients with unknown time of stroke onset were excluded from this study. Patients eligible for intravenous thrombolysis therapy were treated in line with local hospital guidelines. There were no specific treatment recommendations based on CT findings as part of the INSPIRE registry. Written informed consent was obtained from all participants for their information to be collected for the registry, and the INSPIRE study was approved by the local ethics committees in accordance with Australian National Health and Medical Research Council guidelines.

\section{CT protocol}

Acute CT imaging was performed using either 64-, 128-, or 320- detector scanners (GE Lightspeed, Siemens Definition Flash dual source, Philips Brilliance iCT, Siemens sensation 64, Siemens Somatom definition flash and Toshiba Aquilion One).

\section{Imaging analysis}

For the current analyses, all imaging was retrospectively post processed on commercial software MIStar (Apollo Medical Imaging Technology, Melbourne, Australia). The research team used the semi-quantitative tool, ASPECTS system and posterior circulation ASPECTS (pc-ASPECTS), to identify early ischemic change in brain NCCT imaging. ${ }^{20,21}$ For this study, only hypodensity and/or loss of grey-white matter differentiation was rated with ASPECTS; the evaluation of ASPECTS on NCCT images was rated centrally by 2 researchers within INSPIRE trial group (H.K and J.G) in January 2016; all readers were masked to clinical information and other imaging information. Follow-up imaging was not evaluated. All raters were trained using an online tutorial until they were accustomed to the rating procedure. ${ }^{22}$ To control for inter-rater agreement of ASPECTS for identifying EIC, all patients were assessed by two raters for all scores, and disagreements resolved by a third rater.

All CTA images were reviewed by one stroke research fellow. The baseline occlusion was measured according to modified thrombolysis in myocardial infarction (TIMI) parameters from 0 to 3 referring level of distal blood vessel filling. ${ }^{23}$ Collateral status was simply graded based on Miteff system. Miteff system is a three point 
score grading the degree of reconstitution of the cerebral arteries up to the end of its occlusion. ${ }^{24,25}$

\section{Statistical analysis}

The ASPECTS score on pre-treatment NCCT imaging was dichotomized as either ASPECTS $<10$ or ASPECTS $=10$, with the rate of ASPECTS $<10$ representing the detection of EIC. Baseline variables were summarized as frequencies and percentages, or medians and interquartile ranges. These variables (including age, gender, acute NIHSS, baseline TIMI score, and Miteff score) were thought to associate with either or both of the presentation time from symptom onset, or the outcome. Baseline variables were compared between ASPECTS $<10$ and ASPECTS $=10$ cohorts using the Mann-Whitney test for continuous variables and Pearsons's chi-square test for categorical variables. The symptom-to-CT scan times from 0 to 8 hours were stratified by each hour. The research team used a Chisquare test to compare the ASPECTS $<10$ proportions at different time intervals (or time 'groups'). The same analysis was repeated using ASPECTS 0-7 and ASPECTS 8-10 as dichotomized factors, with ASPECTS 0-7 representing widespread hypo-attenuation on $\mathrm{CT}$ image. The variables with the statistical significance were added in logistic regression with dichotomized outcome ASPECTS $<10 /$ ASPECTS=10.

The relationship between dichotomized ASPECTS and symptom-to-CT scan time was investigated using logistic regression with time as a continuous variable. Segmented logistic regression was performed to investigate the potential for time to have an effect on ASPECTS only beyond a certain point. Breakpoints between 60 to 240 minutes by 20 minutes intervals were investigated and the model with the lowest Akaike Information Criterion was selected as the final model. The segments were modelled joined and unjoined at the breakpoint, and likelihood ratio tests were performed to determine which was a better fit for the data. A multivariable logistic regression model was then fit adjusting for age, sex, and NIHSS, TIMI score, Miteff score. Model fit was assessed using Hosmer and Lemeshow goodness of fit test. Odds ratios (ORs) with 95\% confidence interval $(\mathrm{Cl})$ and $P$-values are presented. Statistical significance was defined as $P<0.05$. All statistical analysis was done with STATA statistic software (version 13, Stata Corp., Collage Station, TX, USA) and SAS (version 9.4, SAS Institute, Cary, NC, USA).

\section{Results}

\section{Study population}

The INSPIRE database comprised of 1,329 patients eligible for this study, of which 875 patients had ASPECTS=10, 454 patients had ASPECTS $<10$. Baseline characteristics are described in Table 1.

ASPECTS $<10$ patients were younger than ASPECTS $=10$ patients (median [years], interquartile range [IOR]: $70,60-80$ vs. $74,65-81, P<0.001)$, had higher baseline NIHSS (median, IQR: $14,8-18$ vs. $12,8-15, P<0.001)$, higher symptom-to-CT time (median of minutes, IQR: $145,92-100$ vs. $131,85-161$, $P<0.001$ ), lower TIMI score (median, IQR: $2,0-3$ vs. 3, 1-3, $P<0.001$ ), and lower Miteff score (median, IQR: 2, 1-3 vs. 3, $1-3, P<0.001)$. ASPECTS $0-7$ patients were younger than ASPECTS 8-10 patients (median, IQR: 70, 60-79 year vs. 74, 6481 year, $P=0.009$ ), had higher baseline NIHSS (median, IQR: 15, $9-18$ vs. $12,8-16, P<0.001$ ), higher symptom-to-CT time (median of minutes, IQR: $153,92-219$ vs. $133,87-163, P<0.001$ ), lower TIMI score (median, IQR: 2, 0-3 vs. 2, 1-3, $P=0.006$ ), and lower Miteff score (median, IQR: 2, 1-3 vs. 3, 1-3, $P=0.047$ ).

Within hourly intervals, the proportion of patients with ASPECTS $<10$ increased with increasing delay from symptom-toCT scan time; the change was similar for patients of ASPECTS

Table 1. Baseline characteristics of patients by dichotomized ASPECTSs

\begin{tabular}{|c|c|c|c|c|c|c|}
\hline & ASPECTS $<10$ & ASPECTS $=10$ & $P$ & ASPECTS 0-7 & ASPECTS 8-10 & $P$ \\
\hline No & $454(34)$ & $875(66)$ & & $182(14)$ & $1,147(86)$ & \\
\hline Gender (female) & $213(35)$ & 403 (65) & 0.766 & $84(14)$ & $532(86)$ & 0.954 \\
\hline Age & $70(60-80)$ & $74(65-81)$ & $<0.001$ & $70(60-79)$ & 74 (64-81) & 0.009 \\
\hline Baseline NIHSS & 14 (8-18) & $12(8-15)$ & $<0.001$ & $15(9-18)$ & $12(8-16)$ & $<0.001$ \\
\hline Symptom-to-CT time & $145(92-200)$ & $131(85-161)$ & $<0.001$ & 153 (92-219) & 133 (87-163) & $<0.001$ \\
\hline TIMI score & $2(0-3)$ & $3(1-3)$ & $<0.001$ & $2(0-3)$ & $2(1-3)$ & 0.006 \\
\hline Miteff score & $2(1-3)$ & $3(1-3)$ & $<0.001$ & $2(1-3)$ & $3(1-3)$ & 0.047 \\
\hline
\end{tabular}

Values are presented as number (\%) or medians with interquartile range.

ASPECTS, the Alberta Stroke Program Early Computed Tomography Score; NIHSS, National Institutes of Health Stroke Scale; CT, computed tomography; TIMI, thrombolysis in myocardial infarction. 

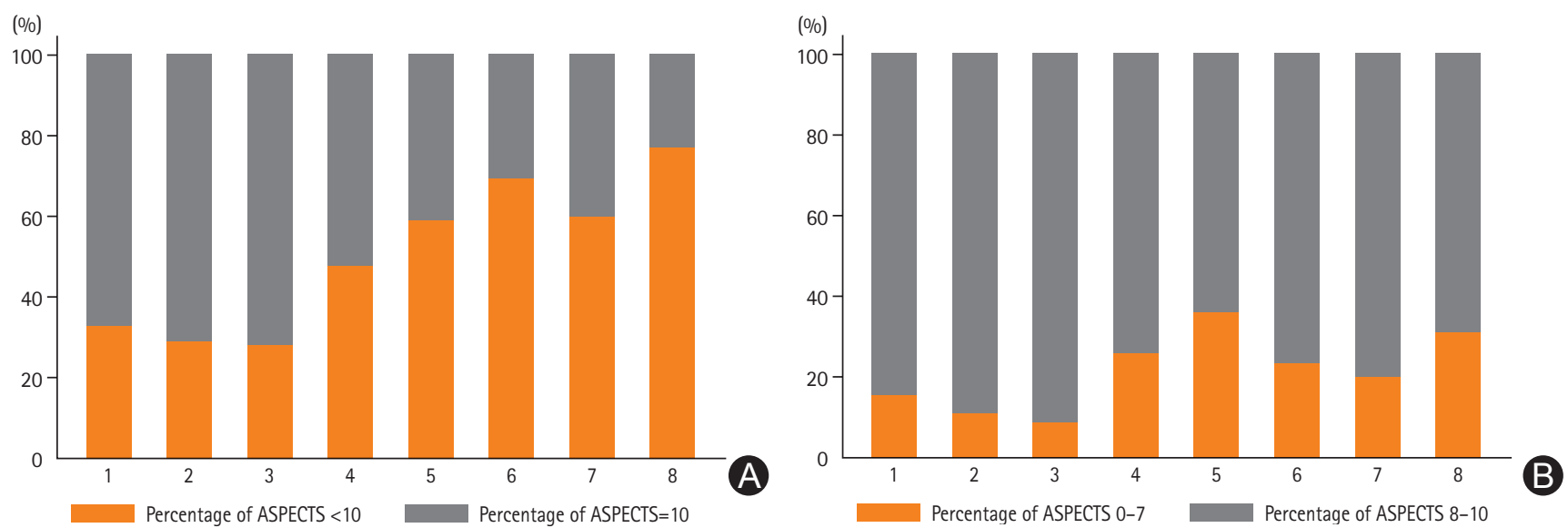

Figure 1. (A) NCCT ASPECTS $<10$ detectable rates (\%) in relation to time after symptom onset. (B) NCCT ASPECTS 0-7 detectable rates (\%) in relation to time after symptom onset. The symptom-to-CT scan times from 0 to 8 hours on X axis were stratified by each hour time. NCCT, non-contrast brain computed tomography; ASPECTS, the Alberta Stroke Program Early Computed Tomography Score.

Table 2. Odds ratio for the effect of time on binary ASPECTS before and after 3 hours

\begin{tabular}{|c|c|c|c|c|c|c|c|c|}
\hline \multirow{2}{*}{ Death } & \multicolumn{3}{|c|}{ Per 1 minute increase } & \multirow{2}{*}{$P$} & \multicolumn{3}{|c|}{ Per 30 minute increase } & \multirow{2}{*}{$P$} \\
\hline & Estimate & $\mathrm{OR}$ & $95 \% \mathrm{Cl}$ & & Estimate & $\mathrm{OR}$ & $95 \% \mathrm{Cl}$ & \\
\hline Effect of time $<3$ hours & -0.002 & 1.00 & (0.99 to 1.00$)$ & 0.266 & -0.053 & 0.95 & (0.86 to 1.04$)$ & 0.266 \\
\hline Effect of time $\geq 3$ hours & 0.006 & 1.01 & (1.00 to 1.01$)$ & 0.002 & 0.182 & 1.20 & (1.07 to 1.34$)$ & 0.002 \\
\hline Effect of time $<3$ hours after adjusted & -0.001 & 1.00 & (0.99 to 1.00 ) & 0.569 & -0.024 & 0.97 & (0.87 to 1.08 ) & 0.569 \\
\hline Effect of time $\geq 3$ hours after adjusted & 0.006 & 1.01 & (1.00 to 1.01$)$ & 0.012 & 0.184 & 1.16 & (1.03 to 1.31$)$ & 0.012 \\
\hline
\end{tabular}

ASPECTS, the Alberta Stroke Program Early Computed Tomography Score; OR, odds ratio; $\mathrm{Cl}$, confidence interval.

0-7 (Figure 1). Among patients who received a CT scan within the first 3 hours after symptom onset, 30\% were rated ASPECTS $<10$. Exploratory chi-square analysis showed that there was no significant difference between hourly groups within the first 3 hours of symptom onset $(P=0.534)$. Chi-square analysis showed the ASPECTS $<10$ rates in the $0-3$ hours group was significantly lower than 4-8 hours group (311/1,066, 29\% vs. $143 / 263,54 \%, P<0.001)$. A significant difference of ASPECTS $0-7$ rates was also found between the $0-3$ hours group and 4-8 hours group (chi-square statistic 110/1,066, 10\% vs. 72/263, 27\%, $P<0.001)$.

Logistic regression was performed to quantify the effect of time on the odds of ASPECTS $<10$. The segmented logistic regression model that best fit the data included a breakpoint at 3 hours with un-joined segments. Zero to three hours after symptom onset, there was no effect of time on odds of CT change (OR 1.00, 95\% Cl 0.99 to 1.00, $P=0.266$, Table 2). After three hours from symptom onset, the odds of ASPECTS $<10$ were significantly related to increasing time from symptom onset to CT. The odds of ASPECTS $<10$ after three hours increased $1 \%(\mathrm{OR}=1.01)$ per 1 minute of time $(95 \% \mathrm{Cl} 1.00$ to

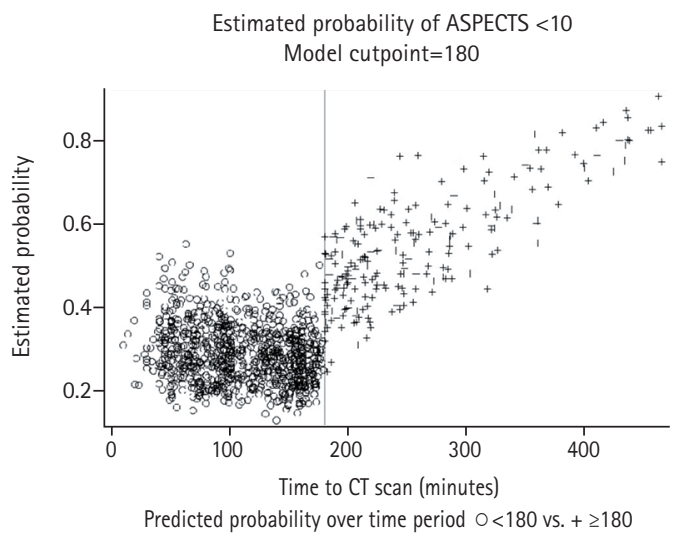

Figure 2. Plot of predicted probability of ASPECTS $<10$ for each patient versus time to $\mathrm{CT}$ scan from the adjusted model. CT, computed tomography; ASPECTS, the Alberta Stroke Program Early Computed Tomography Score.

1.01, $P=0.002$, Table 2). A segmented regression was repeated, adjusting for age, gender, NIHSS, baseline TIMI score, and Miteff score as potential confounders of the relationship between CT change (ASPECTS <10) and time. Prior to three hours the adjusted OR remained non-significant at $1.00(95 \% \mathrm{Cl}$ 
[0.99 to 1.00], $P=0.569$ ), and after 3 hours the adjusted OR per minute was 1.01 (95\% $\mathrm{Cl}[1.00$ to 1.01$], P=0.012)$. The probability of ASPECTS $<10$ for all patients as predicted by the adjusted model, was plotted against time to CT scan (Figure 2).

\section{Discussion}

We have identified that although $30 \%$ of patients have EIC on acute NCCT within the first three hours after symptom onset, there was no significant relationship between time from symptom onset and a decline in ASPECTS within this 3-hour window. There was however, a significant relationship between ASPECTS decline and time for patients imaged beyond three hours from symptom onset. We identified that the odds of an ASPECTS score less than 10 increased by $1 \%$ with every min after stroke onset after 3 hours. A possible explanation for the finding that there was no significant decline in ASPECTS scores in the 0-3 hour time window may suggest inaccurate time of onset in the $30 \%$ of patients with early ischemic change. Alternately it may be that the group of patients with very poor collateral flow, and hence rapid development of infarction with visible oedema, can be identified by the rapid development of ischemic changes, detectable using ASPECTS. ${ }^{26,27}$

The deterioration of collateral quality after stroke onset was strongly associated with rapid ischemic change on NCCT for the patients without reperfusion. In our study, we performed logistic regression with collateral status as a single predictor, which showed that collateral status as measured with the Miteff score was related to early ischemic change on NCCT within the first 3 hours of stroke $(O R=0.847, P=0.005)$. The possible explanation is that baseline poor collateral flow leads to more severe ischemia, which in turn, leads to movement of edema fluid within the first 3 hours of stroke. ${ }^{28,29}$

We have shown that beyond the first 3 hours of stroke onset, the hypo-attenuation sign on NCCT is likely to represent the time-dependent tissue pathophysiology of ischemia rather than disease severity. This finding is supported by animal models which have identified that when residual cerebral blood flow is below $13 \mathrm{~mL} / 100 \mathrm{~g} / \mathrm{min}$ adenosine triphosphate (ATP) depletion can be observed within 30 minutes. ${ }^{30}$ ATP depletion leads to cytotoxic and ionic edema when $\mathrm{Na}$ and water have a net inflow into the cell following electrochemical and ion concentration gradients. Consequently, prolonged vasogenic edema may result in a continuous net water uptake in ischemic tissue, which becomes visible to the human eye on NCCT after 3 hours from ischemia onset. ${ }^{26}$ The aim of this paper is not to alter clinical guidelines or to alter clinical decision making but to explore the possible mechanism behind the appearance of ASPECTS lesions. ${ }^{31}$ The time relationship between the appearance of ASPECTS lesions suggests that more advanced imaging such as angiography or perfusion imaging is a more suitable to aid for clinician decision making.

The main limitation of our study is the low inter-rater agreement for the ASPECTS method which can affect the detection of acute ischemic lesion on NCCT imaging. ${ }^{32}$ However two reviewers assessed all scans and a third reviewer resolved any disagreement. Second, the evaluation of time course of EIC on NCCT is based on NCCT scan at single time point. This might not substantially affect the association between time intervals and EIC on NCCT in our study with a large cohort of patients. Third, in this study white matter changes did not result in an ASPECT point, however we acknowledge that white matter disease may result in a more rapid progression of edema detected using ASPECTS. ${ }^{33}$ Further studies are required to discover the underlying pathophysiological mechanism of early ischemic change on NCCT within first 3 hours.

\section{Conclusions}

Our data suggests that in the 0-3 hour time window from symptom onset, a decline in ASPECTS is not associated with time. However, in the extended time window, a significant relationship between ASPECTS decline and time was evident. This may suggest that early ischemic change with the 0-3 hour time window may be difficult to detect using NCCT alone due either to more subtle changes in density or a protective effect of collateral circulation that is time dependent. These results may also indicate that the presence of lesion on NCCT within three hours of symptom onset for an individual patient may represent an inaccurate reported time of symptom onset. However this will require replication of our result, preferably in a study using serial NCCT imaging.

\section{References}

1. Jauch EC, Saver JL, Adams HP Jr, Bruno A, Connors JJ, Demaerschalk BM, et al. Guidelines for the early management of patients with acute ischemic stroke a guideline for healthcare professionals from the American Heart Association/ American Stroke Association. Stroke 2013;44:870-947.

2. Lees KR, Bluhmki E, von Kummer R, Brott TG, Toni D, Grotta JC, et al. Time to treatment with intravenous alteplase and outcome in stroke: an updated pooled analysis of ECASS, ATLANTIS, NINDS, and EPITHET trials. Lancet 2010;375:1695-1703. 
3. Hacke W, Donnan G, Fieschi C, Kaste M, von Kummer R, Broderick JP, et al. Association of outcome with early stroke treatment: pooled analysis of ATLANTIS, ECASS, and NINDS rt-PA stroke trials. Lancet 2004;363:768-774.

4. Patel SC, Levine SR, Tilley BC, Grotta JC, Lu M, Frankel M, et al. Lack of clinical significance of early ischemic changes on computed tomography in acute stroke. JAMA 2001;286:2830-2838.

5. Hacke W, Kaste $M$, Fieschi $C$, von Kummer $R$, Davalos A, Meier $D$, et al. Randomised double-blind placebo-controlled trial of thrombolytic therapy with intravenous alteplase in acute ischaemic stroke (ECASS II). Second European-Australasian Acute Stroke Study Investigators. Lancet 1998;352:1245-1251.

6. Hacke W, Kaste M, Bluhmki E, Brozman M, Dávalos A, Guidetti $D$, et al. Thrombolysis with alteplase 3 to 4.5 hours after acute ischemic stroke. N Engl J Med 2008;359:1317-1329.

7. Hacke W, Kaste M, Fieschi C, Toni D, Lesaffre E, Von Kummer $\mathrm{R}$, et al. Intravenous thrombolysis with recombinant tissue plasminogen activator for acute hemispheric stroke: the European Cooperative Acute Stroke Study (ECASS). JAMA 1995;274:1017-1025.

8. Clark WM, Wissman S, Albers GW, Jhamandas JH, Madden $K P$, Hamilton S. Recombinant tissue-type plasminogen activator (Alteplase) for ischemic stroke 3 to 5 hours after symptom onset. The ATLANTIS study: a randomized controlled trial. Alteplase Thrombolysis for Acute Noninterventional Therapy in Ischemic Stroke. JAMA 1999;282:2019-2026.

9. Davis SM, Donnan GA, Parsons MW, Levi C, Butcher KS, Peeters $A$, et al. Effects of alteplase beyond $3 \mathrm{~h}$ after stroke in the Echoplanar Imaging Thrombolytic Evaluation Trial (EPITHET): a placebo-controlled randomised trial. Lancet Neurol 2008;7:299-309.

10. Yoo AJ, Berkhemer OA, Fransen PSS, van den Berg LA, Beumer $D$, Lingsma HF, et al. Effect of baseline Alberta Stroke Program Early CT score on safety and efficacy of intra-arterial treatment: a subgroup analysis of a randomised phase 3 trial (MR CLEAN). Lancet Neurol 2016;15:685-694.

11. Kucinski T, Väterlein O, Glauche V, Fiehler J, Klotz E, Eckert B, et al. Correlation of apparent diffusion coefficient and computed tomography density in acute ischemic stroke. Stroke 2002;33:1786-1791.

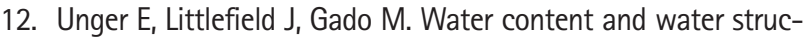
ture in CT and MR signal changes: possible influence in detection of early stroke. AJNR Am J Neuroradiol 1988;9:687-691.

13. Kemmling A, Flottmann F, Forkert ND, Minnerup J, Heindel W, Thomalla G, et al. Multivariate dynamic prediction of ischemic infarction and tissue salvage as a function of time and degree of recanalization. J Cereb Blood Flow Metab
2015;35:1397-1405.

14. Simard JM, Kent TA, Chen M, Tarasov KV, Gerzanich V. Brain oedema in focal ischaemia: Molecular pathophysiology and theoretical implications. Lancet Neuro/ 2007;6:258-268.

15. Symon L, Branston NM, Chikovani O. Ischemic brain edema following middle cerebral artery occlusion in baboons: relationship between regional cerebral water content and blood flow at 1 to 2 hours. Stroke 1979;10:184-191.

16. Dzialowski I, Weber J, Doerfler A, Forsting M, von Kummer R. Brain tissue water uptake after middle cerebral artery occlusion assessed with CT. J Neuroimaging 2004;14:42-48.

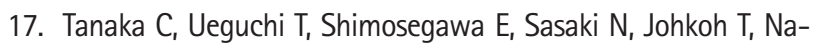

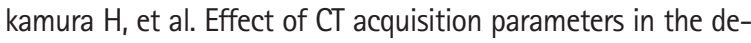
tection of subtle hypoattenuation in acute cerebral infarction: a phantom study. AJNR Am J Neuroradiol 2006;27:40-45.

18. Dzialowski I, Klotz E, Goericke S, Doerfler A, Forsting M, von Kummer R. Ischemic brain tissue water content: CT monitoring during middle cerebral artery occlusion and reperfusion in rats. Radiology 2007;243:720-726.

19. Hara $H$, Muraishi $H$, Matsuzawa $H$, Inoue $T$, Nakajima $Y$, Satoh

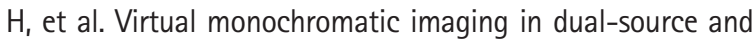
dual-energy CT for visualization of acute ischemic stroke. $J$ Korean Phys Soc 2015;67:103-107.

20. Barber PA, Demchuk AM, Zhang J, Buchan AM. Validity and reliability of a quantitative computed tomography score in predicting outcome of hyperacute stroke before thrombolytic therapy. Aspects study group. Alberta stroke programme early CT score. Lancet 2000;355:1670-1674.

21. Tei H, Uchiyama $S$, Usui T, Ohara K. Posterior circulation ASPECTS on diffusion-weighted MRI can be a powerful marker for predicting functional outcome. J Neurol 2010;257:767-773.

22. CT/DWI early ischemic changes. Acute Stroke Imaging Standardization Group. http://asist.umin.jp/index.htm. 2015. Accessed January 23, 2016.

23. IMS II Trial Investigators. The interventional management of stroke (IMS) II study. Stroke 2007;38:2127-2135.

24. Miteff F, Levi CR, Bateman GA, Spratt N, McElduff P, Parsons $\mathrm{MW}$. The independent predictive utility of computed tomography angiographic collateral status in acute ischaemic stroke. Brain 2009;132:2231-2238.

25. Bivard A, Levi C, Krishnamurthy V, McElduff P, Miteff F, Spratt $\mathrm{NJ}$, et al. Perfusion computed tomography to assist decision making for stroke thrombolysis. Brain 2015;138:1919-1931.

26. Somford DM, Marks MP, Thijs VN, Tong DC. Association of early CT abnormalities, infarct size, and apparent diffusion coefficient reduction in acute ischemic stroke. AJNR Am J Neuroradiol 2004;25:933-938. 
27. Roberts $H C$, Dillon WP, Furlan $A J$, Wechsler LR, Rowley $H A$, Fischbein NJ, et al. Computed tomographic findings in patients undergoing intra-arterial thrombolysis for acute ischemic stroke due to middle cerebral artery occlusion: results from the PROACT II trial. Stroke 2002;33:1557-1565.

28. Vella J, Zammit C, Di Giovanni G, Muscat R, Valentino M. The central role of aquaporins in the pathophysiology of ischemic stroke. Front Cell Neurosci 2015;9:108.

29. Takagi K, Ginsberg MD, Globus MY, Dietrich WD, Martinez E, Kraydieh $\mathrm{S}$, et al. Changes in amino acid neurotransmitters and cerebral blood flow in the ischemic penumbral region following middle cerebral artery occlusion in the rat: correlation with histopathology. J Cereb Blood Flow Metab 1993;13:575-585.

30. Bivard A, Spratt N, Levi CR, Parsons MW. Acute stroke throm- bolysis: time to dispense with the clock and move to tissue-based decision making? Expert Rev Cardiovasc Ther $2011 ; 9: 451-461$

31. Fyfe I. Stroke: CT identifies patients in stroke treatment window. Nat Rev Neurol 2017;13:4-5.

32. van Seeters T, Biessels GJ, Niesten JM, van der Schaaf IC, Dankbaar JW, Horsch AD, et al. Reliability of visual assessment of non-contrast CT, CT angiography source images and CT perfusion in patients with suspected ischemic stroke. PLoS One 2013;8:e75615

33. Wardlaw J, Sandercock P, Dennis M, Starr J. Is breakdown of the blood-brain barrier responsible for lacunar stroke, leukoaraiosis, and dementia? Stroke 2003;34:806-812. 